\title{
Acromegaly as an Expression of a Rare Disease: Description of an Unusual Clinical Case of Carney Complex
}

\author{
Denise Costa ${ }^{1}$, Valeria Mercuri ${ }^{1}$, Tania D'Amico ${ }^{1}$, Giulia Bassotti ${ }^{1}$, Carlo Moroni ${ }^{2}$, \\ Patrizia Gargiulo ${ }^{1}$
}

${ }^{1}$ Department of Experimental Medicine, Endocrinology-Pituitary Disease, “Sapienza” University of Rome, Rome, Italy

${ }^{2}$ Department of Cardiovascular Science, "Sapienza" University of Rome, Rome, Italy

Email: denise.costa@libero.it, v.mercuri79@gmail.com,damico.ta@gmail.com,g_bassotti@yahoo.it, carlo.moroni@uniroma1.it, patrizia.gargiulo@uniroma1.it

How to cite this paper: Costa, D., Mercuri, V., D’Amico, T., Bassotti, G., Moroni, C. and Gargiulo, P. (2020) Acromegaly as an Expression of a Rare Disease: Description of an Unusual Clinical Case of Carney Complex. Case Reports in Clinical Medicine, 9, 59-66.

https://doi.org/10.4236/crcm.2020.92010

Received: December 14, 2019

Accepted: February 4, 2020

Published: February 7, 2020

Copyright $\odot 2020$ by author(s) and Scientific Research Publishing Inc. This work is licensed under the Creative Commons Attribution International License (CC BY 4.0).

http://creativecommons.org/licenses/by/4.0/

\section{(c) (i) Open Access}

\begin{abstract}
Introduction: The Carney complex (CC) is an autosomal dominant syndrome, characterized by multiple endocrine neoplasms, pigmented skin lesions, endocrine hyper-activity and myxomas. Case study: C. E., 71 years old, was addressed to our center in August 1994, following the finding of a sellar enlargement in a skull radiography performed after an accident. A pituitary tomography showed the presence of a lesion extended up to the optic chiasm, a macroadenoma that was treated with transsphenoidal endoscopic surgery. A histological diagnosis of a "GH secreting macroadenoma" was performed. An echocardiographic study highlighted an atrial isolated myxoma that was subsequently removed. A contrast-enhanced abdominal magnetic resonance imaging (MRI) excluded adrenal lesions. In 2001, in relation to relapse of Acromegaly, the patient started therapy with somatostatin analogues which was replaced, in 2008, by Pegvisomant to obtain biochemical control of the disease. Conclusions: At the time of initial screening, it is important not to underestimate the possibility that certain clinical features fall within the context of more complex syndromes. The knowledge of how Acromegaly presents itself in relation to CC and its associated characteristics such as cardiac myxoma or typical skin lesions, will help clinicians to timely diagnose this rare disease and treat it appropriately.
\end{abstract}

Keywords

Acromegaly, Carney, Myxomas, Rare, Syndrome

\section{Introduction}

Carney Complex (CC) was described by Carney for the first time in 1985. CC is 
also known as NAME (Nevi, Atrial myxomas, Myxoid neurofibromas and Ephelides) or LAMB (Lentigines, Atrial myxomas, Mucocutaneous myxomas, Blue nevi), and is a rare autosomal dominant disease characterized by pigmented skin lesions in typical locations (lips, conjunctiva, genital mucosa) and neoplastic manifestations of the endocrine system and other organs (myxomas and schwannomas).

The most common endocrine manifestations include Primary Pigmented Nodular Adrenocortical Disease (PPNAD), a rare cause of ACTH-independent Cushing's syndrome; Large-Cell Calcifying Sertoli Cell Tumor (LCCSCT), a rare testicular tumor; GH- or prolactin-secreting pituitary adenomas; benign or malignant thyroid nodules [1] [2] [3].

In approximately half $(40.7 \%)$ of the known Carney Complex patients, the tumor suppressor gene PRKAR1A, coding for the regulatory subunit type 1-alpha of protein kinase A, showed mutations at the 17q22-24 locus.

Inactivating mutations of PRKAR1A in CC lead to a loss-of-function of the regulatory subunits, therefore to constitutive activation of the cyclic adenosine 3',5'-monophosphate (cAMP)/protein kinase A (PKA) signaling pathway, which results in both proliferative and secretory over activities in endocrine cells.

Another locus, at chromosome $2 \mathrm{p} 16$, is sometimes involved in the molecular pathogenesis of Carney Complex tumors, as demonstrated by the multiple genetic changes that could occur to it, including non-sense substitutions of single bases, small ( $<15 \mathrm{bp})$ deletions/insertions, combined rearrangements, and large deletions that affect most exons and sometimes the entire gene locus. More recently, other mutations have also been described, including defects of the PRKACA and PKRAC genes [4] [5] [6].

Because of the remarkable genetic heterogeneity, it is recommended to use uniform diagnostic criteria [Table 1], with the diagnosis being based on the presence of two or more major clinical criteria. If the patient shows a germinal mutation of PRKAR1A and/or a CC affected first-degree relative, a single clinical manifestation suffices to establish the diagnosis [2].

The knowledge of how Acromegaly presents itself in relation to CC and its associated characteristics such as cardiac myxoma or typical skin lesions, will help clinicians to timely diagnose this rare disease and treat it appropriately.

\section{Case Study}

C. E., 71 years old, was addressed to our center in August 1994, following the finding of a sellar enlargement in a skull radiography performed after an accident. The patient traced the onset of his symptomatology to 1991, when he noticed an increase in the shoe size, visual impairment in the left eye and hyperhidrosis. The physical examination revealed several cutaneous myxomas [Figure 1] on the chest and an ultrasound investigation of the neck demonstrated multi nodular goiter.

A pituitary tomography showed a lesion extended up to the optic chiasm, suggestive of a pituitary macroadenoma [Figure 2]. 
Table 1. Diagnostic criteria for CC Stratakis et al. ${ }^{2}$

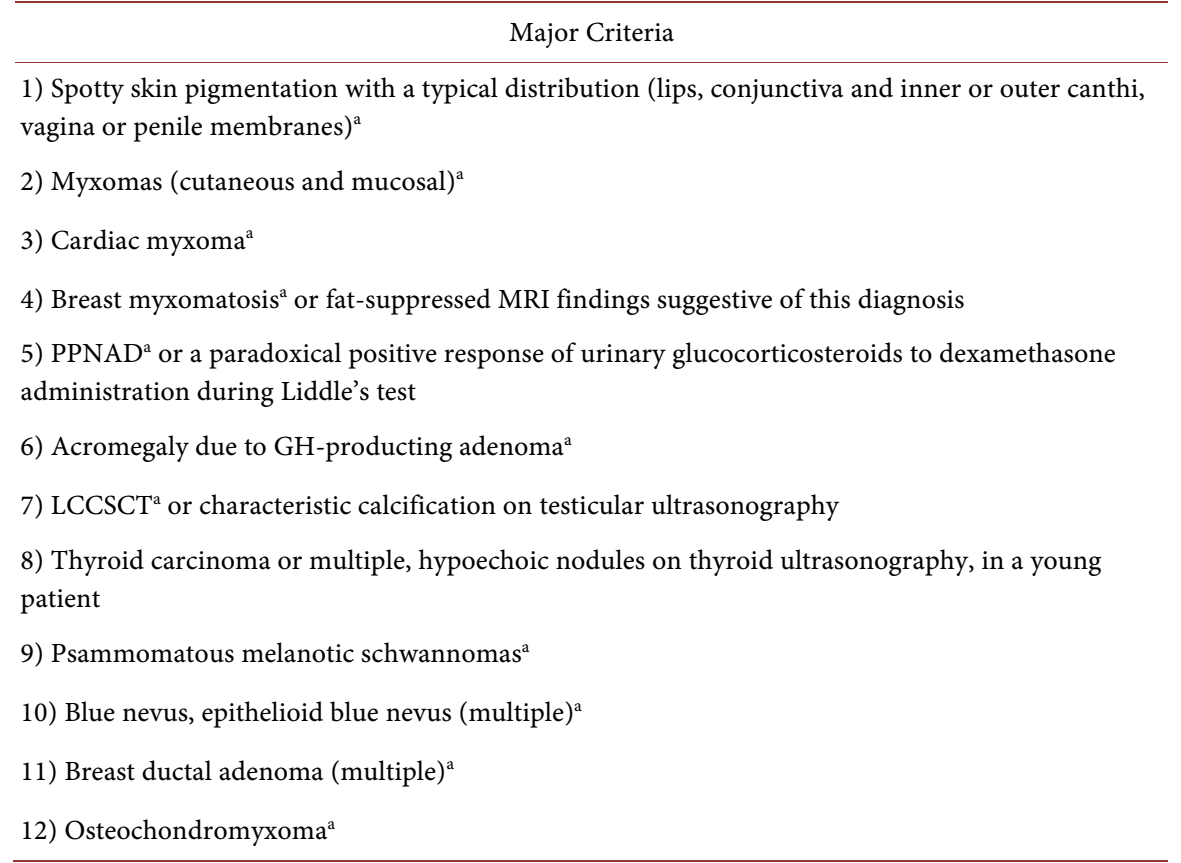

Supplemental criteria: 1) Affected first-degree relative; 2) Inactivating mutation of the PRKAR1A gene. a. Histological confirmation required.

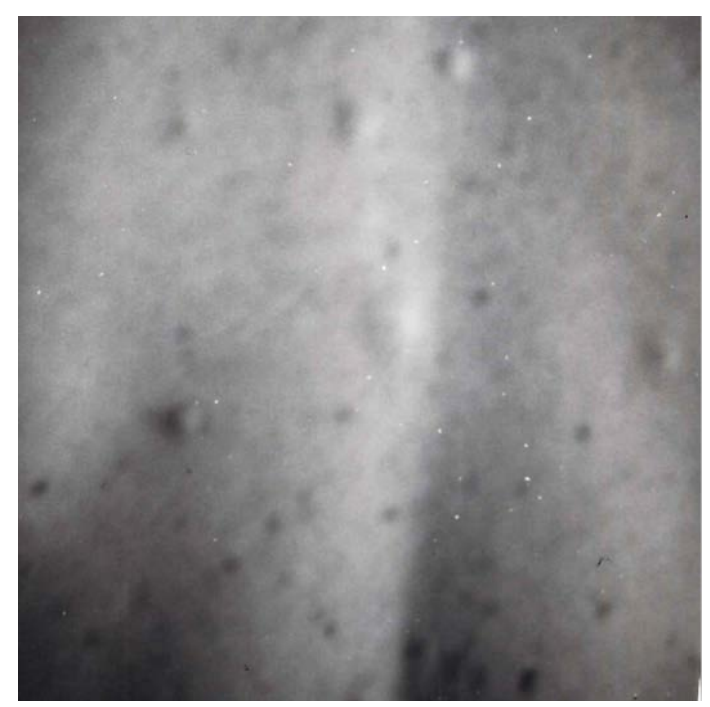

Figure 1. Cutaneous myxomas.

Biochemical and hormonal evaluations demonstrated high values of blood $\mathrm{GH}$ concentrations (40 ng/ml). A transsphenoidal endoscopic surgery was then performed in August 1996. The postoperative period was complicated by a severe dyspnea attributed to obstructive macroglossia. The histologic examination led to the diagnosis of "GH-secreting pituitary macroadenoma".

Echocardiographic controls, performed to evaluate the acromegalic comorbidity, highlighted an atrial isolated myxoma, subsequently surgically removed in October 1999 [Figure 3]. 


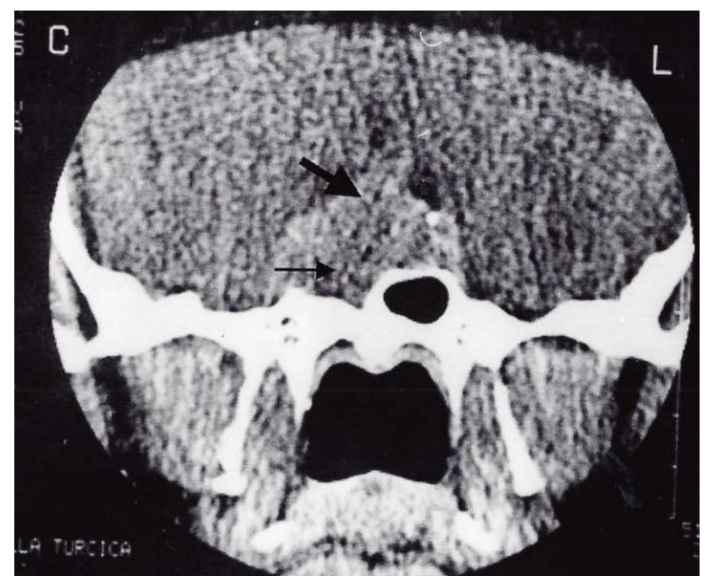

Figure 2. Pituitary macroadenoma.

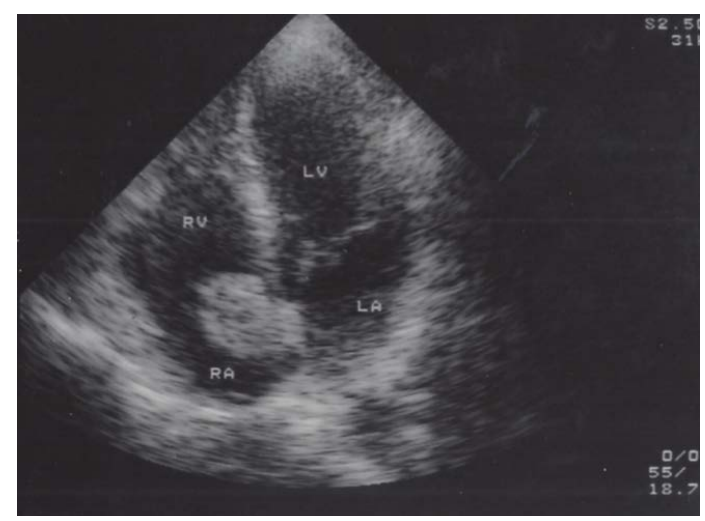

Figure 3. Atrial isolated myxoma.

An ultrasound and a contrast-enhanced abdominal magnetic resonance imaging (MRI) excluded the presence of adrenal lesions.

In 2001, due to relapse of Acromegaly, the patient started therapy with somatostatin analogues (SMS), which was replaced, in 2008, with Pegvisomant in order to obtain the biochemical control of the disease.

In 2008, the patient underwent a pituitary MRI that did not show contrast-enhanced areas referable to acromegalic disease recurrence.

Following the worsening of drowsiness, snoring and anxiety, with consequent decline in quality of life, the patient underwent a cardio-respiratory monitoring which revealed a severe obstructive sleep apnea syndrome (OSAS). The apnea-hypopnea index (AHI) was 38.4 (n.v. < 5). Therefore C-PAP therapy was prescribed but the patient was not compliant. He died in 2017 due to a pulmonary embolism.

As for the familiar history, it was also to be taken into account that the patient, at the time of the diagnosis, had two children (a 25 years old female and a 30 years old male) and three grandchildren in the infant age.

\section{Discussion}

In the literature, there are 750 known patients with CC, including Caucasians, 
African Americans, and Asians from all continents. Approximately $70 \%$ of CC cases had an affected relative, whereas the remaining 30\% carried a de novo germline mutation [3]. There is a significant variability in clinical manifestations of the disease, also among members of the same family, which could lead to different diagnoses, although we are in the presence of the same pathology. Therefore, a genetic study and/or a prolonged follow-up is highly recommended in family members.

Our patient presented 4 major criteria for the diagnosis of Carney Complex: cutaneous myxoma, cardiac myxoma, GH pituitary macroadenoma, multinodular goiter.

However, it should be noted that he did not show any signs of PPNAD (which occurs in $25 \%$ of patients) and that the diagnosis was performed at an older age than the average described in literature. Moreover, the single cardiac myxoma did not occur again after surgery. The genetic origin of the complex could not be definitively determined, because the patient and his family refused to undergo the genetic test.

For more than 20 years, we have performed careful clinical, imaging and biochemical screenings of all his first- and second-degree relatives and we have not obtained pathological data. In the light of the above, our patient seems to be considered "a sporadic case".

However, the late onset of the disease in our patient could lead, in the future, to the appearance of some manifestations in his relatives.

In view of the recent discovery of mutations in the PRKAR1A gene, genetic screening should be recommended to our patient and his relatives, as well as an estimate of penetrance of the mutation would be desirable.

In reference to acromegaly within the more complex framework of CC, its progression is generally slower. The incidence of GH-secreting pituitary tumors has been estimated to be less than $15 \%$, although $80 \%$ of cases have asymptomatic increases in GH and IGF1 levels [7].

This is likely due to a certain degree of somatotroph hyperplasia which suggests that PRKAR1A mutations may lead to GH hyperplasia, which in turn may sometimes result, albeit not systematically, in the development of a GH-secreting micro or macro adenoma [8].

Less is known about the efficacy of medical treatment of acromegaly in the setting of CC. Previous work suggested that when the cAMP signaling pathway was overactivated in somatotroph tumors, through mutation of the gsp oncogene, a greater inhibition of the GH secretion was observed under octreotide, as compared to non-mutated tumors [9] [10]. In CC, a roughly similar molecular mechanism is likely to occur as the PRKAR1A mutation ultimately leads to a sustained activation of both c-AMP response element-binding protein (CREB) and gene transcription. However, in our patient, comparison of complications and resistance to SMS occurred, suggesting that other mechanisms may influence this therapeutic effect.

Hypersecretion of prolactin, albeit mild, appears to be frequent in Carney 
Complex patients and may be associated with high levels of IGF-I in some, but not all, patients [11].

Conversely, among the most frequent endocrine tumors, PPNAD occurs in $26 \%$ of patients [1] [12] [13]. This value, however, significantly underestimates the real incidence of PPNAD among Carney's patients: a biochemical screening performed by dexamethasone stimulation tests revealed the presence of a greater number of patients with subclinical, atypical or sporadic PPNAD. In addition, histologic evidence of PPNAD was found in almost all patients with the complex who underwent autopsy [12] [13]. The choice treatment for PPNAD is bilateral adrenalectomy in order to prevent the devastating long-term effects of hypercortisolism [14].

The finding of thyroid nodules is a very common one in the Carney complex, which includes a spectrum of abnormalities ranging from follicular hyperplasia and/or cystic changes to carcinoma. Ultrasound is useful for the diagnosis and clinical follow-up of these lesions, which are commonly reported as hypoechoic lesions [15].

Finally, even if not present in this case, we recall the rare large calcified Sertoli cell tumor, often multicenter and bilateral [16].

Awareness of CC in urology is important because of the risk of developing testicular and infertility tumors, which makes the transmission of the disease statistically less likely through affected males [17].

The clinical and biochemical screening for the Carney Complex remains the gold standard for diagnosis. The genetic study, at the time, couldn't be used for the diagnosis considering the genetic heterogeneity and the ongoing study of new mutated genes, but it could be used for screening in families in which the mutated genes are known. In relation to the clinical complexity, the patient with Carney Complex, once the diagnosis is established, must annually undergo an ultrasound evaluation of the heart, thyroid, abdomen and gonads, as well as hormonal assays for assessing the hypothalamic-pituitary-adrenal-gonads axis.

\section{Conclusions}

We decided to publish this case to point out that the context of endocrinological clinical features (or pathologies) is fundamental, at least in the initial screening, does not underestimate the possibility of more complex syndromes.

This case report is noteworthy for the clinical features (age of onset and diagnosis, acromegaly resistant to different treatments, absence of PPNAD and testicular tumors and infertility) within the CC but also for the reason that the patient and his relatives were followed for a long time by the same endocrinologist.

After 20 years of observation, the patient did not present other significant pathologies, neither progression of comorbidities of acromegaly nor recurrence of myxoma. The observation of the relatives ( 1 son and 1 daughter and 3 grandchildren) allowed excluding CC.

Failure to carry out the genetic study is a gap, but it will be our commitment to insist again on family members. 


\section{Conflicts of Interest}

The authors declare no conflicts of interest regarding the publication of this paper.

\section{References}

[1] Carney, J.A., Gordon, H., Carpenter, P.C., Shenoy, B.V. and Go, V.L. (1985) The Complex of Myxomas, Spotty Pigmentation, and Endocrine Over-Activity. Medicine (Baltimore), 64, 270-283. https://doi.org/10.1097/00005792-198507000-00007

[2] Stratakis, C.A., Kirschner, L.S. and Carney, J.A. (2001) Clinical and Molecular Features of the Carney Complex: Diagnostic Criteria and Recommendations for Patient Evaluation. The Journal of Clinical Endocrinology \& Metabolism, 86, 4041-4046. https://doi.org/10.1210/jcem.86.9.7903

[3] Correa, R., Salpea, P. and Stratakis, C.A. (2015) Carney Complex: An Update. European Journal of Endocrinology, 173, M85-M97. https://doi.org/10.1530/EJE-15-0209

[4] Kirschner, L.S., Carney, J.A., Pack, S.D., Taymans, S.E., Giatzakis, C., Cho, Y.S., et al. (2000) Mutations of the Gene Encoding the Protein Kinase A Type I-Alpha Regulatory Subunit in Patients with the Carney Complex. Nature Genetics, 26, 89-92. https://doi.org/10.1038/79238

[5] Bosco Schamun, M.B., Correa, R., Graffigna, P., de Miguel, V. and Fainstein Day, P. (2018) Carney Complex Review: Genetic Features. Endocrinología, Diabetes y Nutrición, 65, 52-59. https://doi.org/10.1016/j.endinu.2017.09.006

[6] Casey, M., Vaughan, C.J., He, J., et al. (2001) Mutations in the Protein Kinase A R1alpha Regulatory Subunit Cause Familial Cardiac Myxomas and Carney Complex. Journal of Clinical Investigation, 106, 31-38. https://doi.org/10.1172/JCI10841

[7] Stergiopoulos, S.G., Abu-Asab, M.S., Tsokos, M. and Stratakis, C.A. (2004) Pituitary Pathology in Carney Complex Patients. Pituitary, 7, 73-82. https://doi.org/10.1007/s11102-005-5348-y

[8] Cuny, T., Mac, T.T., Romanet, P., Dufour, H., Morange, I., Albarel, F., et al. (2019) Acromegaly in Carney Complex. Pituitary, 22, 456-466. https://doi.org/10.1007/s11102-019-00974-8

[9] Barlier, A., Gunz, G., Zamora, A.J., Morange-Ramos, I., Figarella-Branger, D., Dufour, H., et al. (1998) Pronostic and Therapeutic Consequences of Gs Alpha Mutations in Somatotroph Adenomas. The Journal of Clinical Endocrinology \& Metabolism, 83, 1604-1610. https://doi.org/10.1210/jcem.83.5.4797

[10] Efstathiadou, Z.A., Bargiota, A., Chrisoulidou, A., Kanakis, G., Papanastasiou, L., Theodoropoulou, A., et al. (2015) Impact of GSP Mutations in Somatotroph Pituitary Adenomas on Growth Hormone Response to Somatostatin Analogs: A Meta-Analysis. Pituitary, 18, 861-867. https://doi.org/10.1007/s11102-015-0662-5

[11] Raff, S.B., Carney, J.A., Krugman, D., Doppman, J.L. and Stratakis, C.A. (2000) Prolactin Secretion Abnormalities in Patients with the "Syndrome of Spotty Skin Pigmentation, Myxomas, Endocrine Overactivity and Schwannomas" (Carney Complex). Journal of Pediatric Endocrinology and Metabolism, 13, 373-379. https://doi.org/10.1515/JPEM.2000.13.4.374

[12] Manipadam, M.T., Abraham, R., Sen, S. and Simon, A. (2011) Primary Pigmented Nodular Adrenocortical Disease. Journal of Indian Association of Pediatric Surgeons, 16, 160-162. https://doi.org/10.4103/0971-9261.86881 
[13] Stratakis, C.A., Sarlis, N., Kirschner, L.S., Carney, J.A., Doppman, J.L., Nieman, L.K., et al. (1999) Paradoxical Response to Dexamethasone in the Diagnosis of Primary Pigmented Nodular Adrenocortical Disease. Annals of Internal Medicine, 131, 585-591. https://doi.org/10.7326/0003-4819-131-8-199910190-00006

[14] Koch, C.A., Bornstein, S.R., Chrousos, G.P. and Stratakis, C.A. (2000) Primary Pigmented Nodular Adrenocortical Dysplasia (PPNAD) within the Scope of Carney Complex as the Etiology of Cushing Syndrome. Medizinische Klinik (Munich), 95, 224-230. https://doi.org/10.1007/PL00002112

[15] Stratakis, C.A., Courcoutsakis, N.A., Abati, A., Filie, A., Doppman, J.L., Carney, J.A., et al. (1997) Thyroid Gland Abnormalities in Patients with the Syndrome of Spotty Skin Pigmentation, Myxomas, Endocrine Overactivity, and Schwannomas (Carney Complex). The Journal of Clinical Endocrinology \& Metabolism, 82, 2037-2043. https://doi.org/10.1210/jcem.82.7.4079

[16] Premkumar, A., Stratakis, C.A., Shawker, T.H., Papanicolaou, D.A. and Chrousos, G.P. (1997) Testicular Ultrasound in Carney Complex: Report of Three Cases. Journal of Clinical Ultrasound, 25, 211-214. https://doi.org/10.1002/(SICI)1097-0096(199705)25:4<211::AID-JCU10>3.0.CO;2$\underline{\mathrm{N}}$

[17] Alleemudder, A. and Pillai, R. (2016) A Case of Carney Complex Presenting as Acute Testicular Pain. Urology Annals, 8, 360-362.

https://doi.org/10.4103/0974-7796.184887 